\title{
Identifying the function of methylated genes in Alzheimer's disease to determine epigenetic signatures: a comprehensive bioinformatics analysis
}

\author{
Md Rezanur Rahman ${ }^{1,2, *}$ (D), Tania Islam², Esra Gov ${ }^{3}$, Julian M.W. Quinn ${ }^{4}$ and \\ Mohammad Ali Moni ${ }^{4,5}$ \\ ${ }^{1}$ Department of Biochemistry and Biotechnology, School of Biomedical Science, Khwaja Yunus Ali University, Enayetpur, \\ Sirajgonj, Bangladesh, ${ }^{2}$ Department of Biotechnology and Genetic Engineering, Faculty of Biological Sciences, Islamic \\ University, Kushtia, Bangladesh, ${ }^{3}$ Department of Bioengineering, Adana Alparslan Turkes Science and Technology University, \\ Adana, Turkey, ${ }^{4}$ Healthy Aging Theme, Garvan Institute of Medical Research, Darlinghurst, NSW, Australia, and ${ }^{5}$ WHO \\ Collaborating Centre on eHealth, School of Public Health and Community Medicine, Faculty of Medicine, The University of \\ New South Wales, Sydney, Australia \\ *Corresponding author: E-mail: rezanur12@yahoo.com
}

(Received 05 November 2020; Revised 23 November 2020; Accepted 24 November 2020)

\begin{abstract}
Gene methylation is one means of controlling tissue gene expression, but it is unknown what pathways influencing Alzheimer's disease (AD) are controlled this way. We compared normal and AD brain tissue data for gene expression (mRNAs) and gene methylation profiling. We identified methylated differentially expressed genes (MDEGs). Protein-protein interaction (PPI) of the MDEGs showed 18 hypermethylated low-expressed genes (Hyper-LGs) involved in cell signaling and metabolism; also 10 hypomethylated highly expressed (Hypo-HGs) were involved in regulation of transcription and development. Molecular pathways enriched in Hyper-LGs included neuroactive ligand-receptor interaction pathways. Hypo-HGs were notably enriched in pathways including hippo signaling. PPI analysis also identified both Hyper-LGs and HypoHGs, as hub proteins. Our analysis of AD datasets identified Hyper-LGs, Hypo-HGs, and transcription factors linked to these genes. These pathways, which may participate in Alzheimer's disease development, may be affected by treatments that influence gene methylation patterns.
\end{abstract}

Keywords: Alzheimer's disease; epigenetics; biomarkers; methylated differentially expressed genes.

\section{Introduction}

Alzheimer's disease $(\mathrm{AD})$ is a neurodegenerative disease characterized by the accumulation of amyloid plaques and neurofibrillary tangles in neuron cells and which is manifested by the gradual development of dementia symptoms, including profound impairment of cognitive abilities (Rahman et al., 2020). The pathobiology of the $\mathrm{AD}$ is complex with genetic and epigenetic events are involved in the disease pathogenesis (Stoccoro \& Coppede, 2018). While the hallmarks of the disease include the accumulation of amyloid plaques and neurofibrillary tangles in the brain (Dunckley et al., 2006), how these are related to $\mathrm{AD}$ development and, indeed, what are the key underlying mechanisms of $\mathrm{AD}$ is uncertain. A number of gene expression profiling studies have been performed comparing neuronal tissues of AD and control patients, including microarray gene expression analysis and array or sequencing-based analyses of bisulfite converted DNA to detect differences in gene methylation levels (Chouliaras et al., 2013; 
Coppieters et al., 2014). The latter identifies and quantifies a key (though not the sole) epigenetic control of gene expression since a methylated gene promoter generally has blocked transcription. However, a combined approach to integrate gene expression and gene methylation data could uncover epigenetic signatures in AD.

Epigenetic mechanisms, including DNA methylation and histone modifications, play a crucial role in the development of the AD (Sanchez-Mut \& Graff, 2015). Therefore, identification of methylateddifferentially expressed genes (MDEGs) and discovering pathways may be useful for the clarification of how these and other mechanisms associated with AD may be controlled. Earlier studies have identified gene signatures in AD (Rahman et al., 2020; Semick et al., 2019). These studies provided gene signatures focused on either gene expression or methylation profiling. To provide an in-depth understanding of the biological mechanisms of $\mathrm{AD}$, a conjoint analysis of gene expression and gene methylation analysis is considered.

\subsection{Objective}

In this study, we performed bioinformatic analysis of gene expression (mRNAs) and DNA methylation data from $\mathrm{AD}$-affected neural tissues to identify differentially expressed genes (DEGs) and differentially methylated genes (DMGs), respectively. We aimed to identify overlapping methylated differentially expressed genes (MDEGs) to provide novel insights in $\mathrm{AD}$ pathogenesis. Our workflow of the analysis is summarized in Fig. 1.

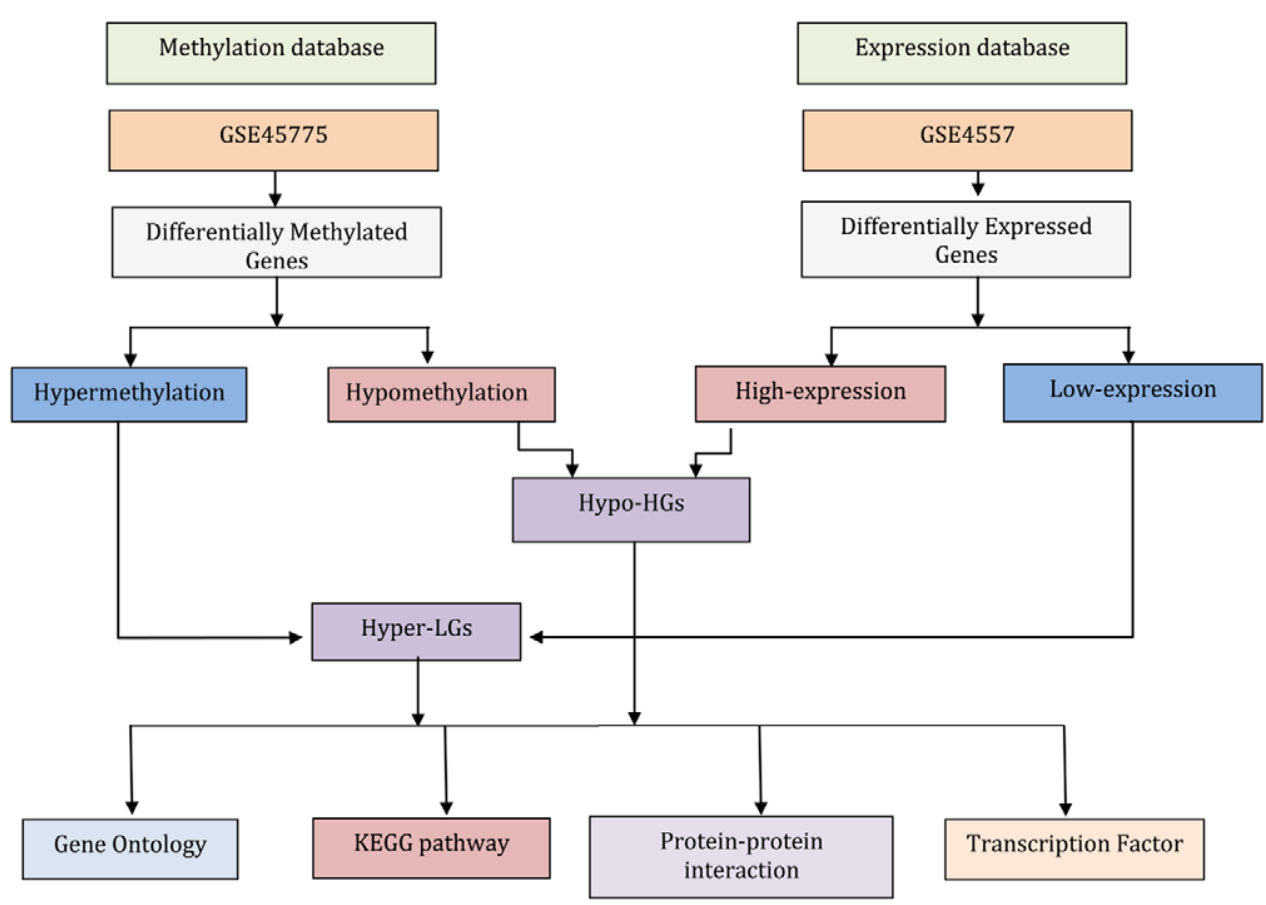

Figure 1. Flowchart describing the data analysis processes in this work. The gene expression data for neurons from postmortem brain tissue from Alzheimer's disease (AD) patients and matched controls were used to identify differentially expressed genes (DEGs). Similarly, genome-wide DNA methylation data of AD compared matched controls were subjected to identify differentially methylated genes (DMGs). Comparing highly expressed genes with hypomethylation genes (i.e., genes that have high expression levels because of a lack of suppression by methylation) identified the genes termed here Hypo-HGs. Similarly, low-expression genes with hypermethylation (suggesting low expression levels due to suppression methylation) identified the genes termed here Hyper-LGs. Then, we annotated Hypo-HGs and Hyper-LGs using Gene Ontology (GO) and KEGG pathway to identify GO and pathway. The protein-protein interaction (PPI) networks of the Hypo-HGs and Hyper-LGs were also investigated to identify hub genes for these networks. Gene-transcription (TF) factor analysis was also performed to detect potential key regulators of the activities of these genes. 


\section{Methods}

\subsection{Acquisition of transcriptomic and DNA methylation datasets}

We utilized mRNA gene expression profiling data (GSE4757) and DNA methylation profiling data (GSE45775) from studies of AD and control samples of brain tissue. These datasets were obtained from the NCBI-GEO database. The GSE4757 mRNA profiling datasets contained 20 samples that consisted of $10 \mathrm{AD}$ tissue samples and 10 non-AD control tissue samples. Samples were obtained from the same patient and the same brain region. Selected neurons containing neurofibrillary tangles and normal neurons from the entorhinal cortex of 10 mid-stage $\mathrm{AD}$ cases via laser capture microdissection were used for gene expression dataset. The methylation microarray data from GSE45775 dataset contained 20 samples that included $15 \mathrm{AD}$ tissues and 5 control samples which consisted of DNA methylation profiling of normal hippocampus and different Alzheimer Braak stages hippocampus samples. The entorhinal cortex is an area of the brain located in the medial temporal lobe which has a central role in neuronal networks that underlie memory functions. Similarly, the hippocampus also plays a key role in memory and knowledge acquisition. It was recently determined that the entorhinal cortex could be a new player for memory formation that works in parallel to the hippocampus (O’Neill et al., 2017). Although data obtained from the two different brain regions were integrated in the present study, both of these brain regions are thought to participate in memory functions.

\subsection{Data processing and identification of differentially expressed genes}

We employed GEO2R web-utility to identify DEGs and differentially methylated genes (DMGs) by comparing $\mathrm{AD}$ samples compared to control. The microarray datasets were processed and normalized in GEO2R. A $p$-value $<0.05$ and $|t|>2$ was considered as the cut-off criteria to identify the DEGs and DMGs. We identified overlapping MDEGs between the GSE4757 and GSE45775 datasets. The mutually common genes between down-regulated and hypermethylation genes were termed as hypermethylatedlowly expressed genes (Hyper-LGs). Similarly, the common genes between upregulated and hypomethylation genes were regarded as hypomethylated-highly expressed genes (Hypo-HGs).

\subsection{Functional and pathway enrichment analysis}

We performed functional annotation of the identified MDEGs via Enrichr (Kuleshov et al., 2016) to detect Gene ontology (GO) terms and KEGG pathways. $p$-value $<0.05$ was considered as statistically significant for enrichment analysis.

\subsection{Protein interactome analysis}

We utilized the STRING database (Szklarczyk et al., 2017) to study the protein-protein interaction (PPI) network for Hyper-LGs and Hypo-HGs the via NetworkAnalyst (Xia et al., 2015). The hubs were selected based on degree $>20$ to identify a high number of interacting hub proteins in the PPI networks.

\subsubsection{Transcription factor analysis}

We have analyzed and identified the regulatory transcription factors (TFs) that interact with MDEGs, suggesting these TFs may regulate the identified MDEGs utilizing the TRNASFAC and JASPAR databases via Enrichr (Kuleshov et al., 2016). A p-value $<0.05$ was considered to designate the statistically significant TFs.

\section{Results}

\subsection{Methylated differentially expressed genes in $A D$}

We analyzed the gene expression and methylation data to identify DEGs or DMGs. We identified overlapping genes, termed here 18 Hyper-LGs, by matching down-regulated DEGs with the 
hypermethylated DMGs; 10 Hypo-HGs were identified by comparing and up-regulated DEGs and hypomethylated DMGs.

To clarify the biological significance of the identified MDEGs, GO enrichment analysis was performed (Table S1). With regard to Hyper-LGs, enriched biological processes (BP) included notably positive regulation of potassium ion transport, and regulation of glucose metabolic process. The enriched GO terms for Hypo-HGs were enriched in BP included positive regulation of transcription.

\subsection{Molecular pathways from epigenetic perspective}

The Hyper-LGs demonstrated enrichment in pathways of nitrogen metabolism, nicotine addiction, neuroactive ligand-receptor interaction, amyotrophic lateral sclerosis (ALS). Hypo-HGs were significantly involved in hippo signaling pathway, cGMP-PKG signaling pathway, alcoholism, TGF-beta signaling pathway (Table 1:).

\subsection{Protein-protein Interaction to identify hub proteins}

We analyzed the PPI of MDEGs. The Hyper-LGs PPI network had 208 nodes and 209 edges (Fig. 2), while the Hypo-HGs network consisted of 542 nodes and 574 edges (Fig. 3). Thetopological analysis showed hub genes for both the Hyper-LGs and Hypo-HGs networks. Hub proteins (TOMM22, TBX5, ANK2, GRIA2, COPS7B, RORA) were detected as Hyper-LGs, while hub proteins (BMP2, GATA4, HDAC11, GGA2, CREB3, RASSF1) were identified for Hypo-HGs.

\subsubsection{Transcription factors of methylated-differentially expressed genes}

The generation of gene products can be regulated at both transcriptional and post-transcriptional levels. The TFs directly regulate the expression (i.e., transcription) of DEGs, thus we sought to detect the TFs that may regulate the MDEGs. Table 2: showed the TFs that regulate the MDEGs.

\section{Discussion}

The development and progression of $\mathrm{AD}$ is the result of complex interplay of epigenetics and genetics mechanisms at multistage. Epigenetic perturbation, especially of DNA methylation, contributes immensely to the pathobiology of AD (Stoccoro \& Coppede, 2018; Kawalia et al., 2017). The identification of potential biomarkers for $\mathrm{AD}$ will not only improve the understanding of how the pathogenesis of $\mathrm{AD}$ is controlled but may also open new avenues of treatment strategies. We identified 18 Hyper-LGs and 10 Hypo-HGs as key gene signature in AD. The enrichment and PPI analysis provided significant pathways and methylated hub genes which may provide novel insights into the pathogenesis of AD. The pathway analysis of Hyper LGs showed enriched ALS pathways (Rusina et al., 2007) in ALS patients may be accompanied by cognitive impairment and existence of neurofibrillary tangles and plaques affecting neurons, (Rusina et al., 2007) suggesting the importance of the identified pathways in AD pathogenesis (Ravetti et al., 2010). Our analysis also showed pathways enriched by Hypo-HGs. Among the pathways, the pre-activation of hippo signaling pathway is associated with neurodegenerative diseases including AD (Mueller et al., 2018). We obtained "alcoholism pathway" enriched by the MDEGs, which probably plays roles in $\mathrm{AD}$ pathogenesis because the alcohol has been found to be involved in neuroinflammation in dementia, suggesting an additional mechanism in neurodegenerative disease. Our analysis identified "TGF-beta signaling pathway" as involved in AD. Increasing evidence suggests that dysregulation of TGF-beta signaling pathway play critical roles in AD (von Bernhardi et al., 2015). In brief, in order to identify new therapeutic targets, exploration of signaling pathways and biomarkers involved in MDEGs will provide new understanding about $\mathrm{AD}$ pathogenesis.

We studied the PPI based on the proteins encoded by the MDEGs. Among the hubs, TOMM22 serve as the main receptor for accumulation of amyloid $\beta(A \beta)$ peptides in $\mathrm{AD}$ (Hu et al., 2018). Previous 
Table 1. Pathway analysis of methylated-differentially expressed genes related to Alzheimer's disease patient samples.

\begin{tabular}{|c|c|c|c|c|}
\hline \multicolumn{2}{|l|}{ Category } & \multirow{2}{*}{$\begin{array}{l}\text { Term } \\
\text { Nitrogen metabolism }\end{array}$} & \multirow{2}{*}{$\begin{array}{l}\text { Adj. } p \text {-value } \\
0.015\end{array}$} & \multirow{2}{*}{$\frac{\text { Genes }}{\text { CA12 }}$} \\
\hline Hyper-LGs & KEGG & & & \\
\hline & & Circadian rhythm & 0.027 & RORA \\
\hline & & Pentose and glucuronate interconversions & 0.030 & $\mathrm{KL}$ \\
\hline & & Nicotine addiction & 0.035 & GRIA2 \\
\hline & & Neuroactive ligand-receptor interaction & 0.036 & GRIA2;ADRA2A \\
\hline & & Endocrine and other factor-regulated calcium reabsorption & 0.042 & $\mathrm{KL}$ \\
\hline & & Cocaine addiction & 0.043 & GRIA2 \\
\hline & & Amyotrophic lateral sclerosis (ALS) & 0.044 & GRIA2 \\
\hline \multirow[t]{22}{*}{ Hypo-HGs } & KEGG & Hippo signaling pathway & 0.002 & BMP2;RASSF1 \\
\hline & & cGMP-PKG signaling pathway & 0.002 & CREB3;GATA4 \\
\hline & & Alcoholism & 0.003 & CREB3;HDAC11 \\
\hline & & Viral carcinogenesis & 0.004 & CREB3;HDAC11 \\
\hline & & RNA polymerase & 0.015 & POLR3E \\
\hline & & Bladder cancer & 0.020 & RASSF1 \\
\hline & & Vasopressin-regulated water reabsorption & 0.021 & CREB3 \\
\hline & & Cocaine addiction & 0.024 & CREB3 \\
\hline & & Pathways in cancer & 0.027 & BMP2;RASSF1 \\
\hline & & Basal cell carcinoma & 0.031 & BMP2 \\
\hline & & Cytosolic DNA-sensing pathway & 0.031 & POLR3E \\
\hline & & Cortisol synthesis and secretion & 0.032 & CREB3 \\
\hline & & Non-small cell lung cancer & 0.032 & RASSF1 \\
\hline & & Amphetamine addiction & 0.033 & CREB3 \\
\hline & & Thyroid hormone synthesis & 0.036 & CREB3 \\
\hline & & Leishmaniasis & 0.036 & CYBA \\
\hline & & Insulin secretion & 0.042 & CREB3 \\
\hline & & TGF-beta signaling pathway & 0.044 & BMP2 \\
\hline & & Prostate cancer & 0.047 & CREB3 \\
\hline & & Aldosterone synthesis and secretion & 0.047 & CREB3 \\
\hline & & Melanogenesis & 0.049 & CREB3 \\
\hline & & Longevity regulating pathway & 0.049 & CREB3 \\
\hline
\end{tabular}

studies showed that the gene ANK2 is involved in AD (Higham et al., 2018). Our analysis also detected $R O R A$ as a hub which is distinctively overexpressed in the hippocampus of AD brain (Acquaah-Mensah et al., 2015). With regard to Hypo-HGs, we detected seven hub proteins, including BMP3, GATA4, HDAC11 and CREB, which have been previously described in brain functions and neurodegenerative diseases. Among these hubs we noted BMP2, the nuclear form of BMP2 has previously been shown to play a role in hippocampus memory formation (Cordner et al., 2017). Transcription factor GATA4 has been shown to be significantly differentially expressed in AD compared to controls (Garranzo-Asensio et al., 2018). It should also be noted that HDAC inhibitors are known to be a potential drug target in AD 


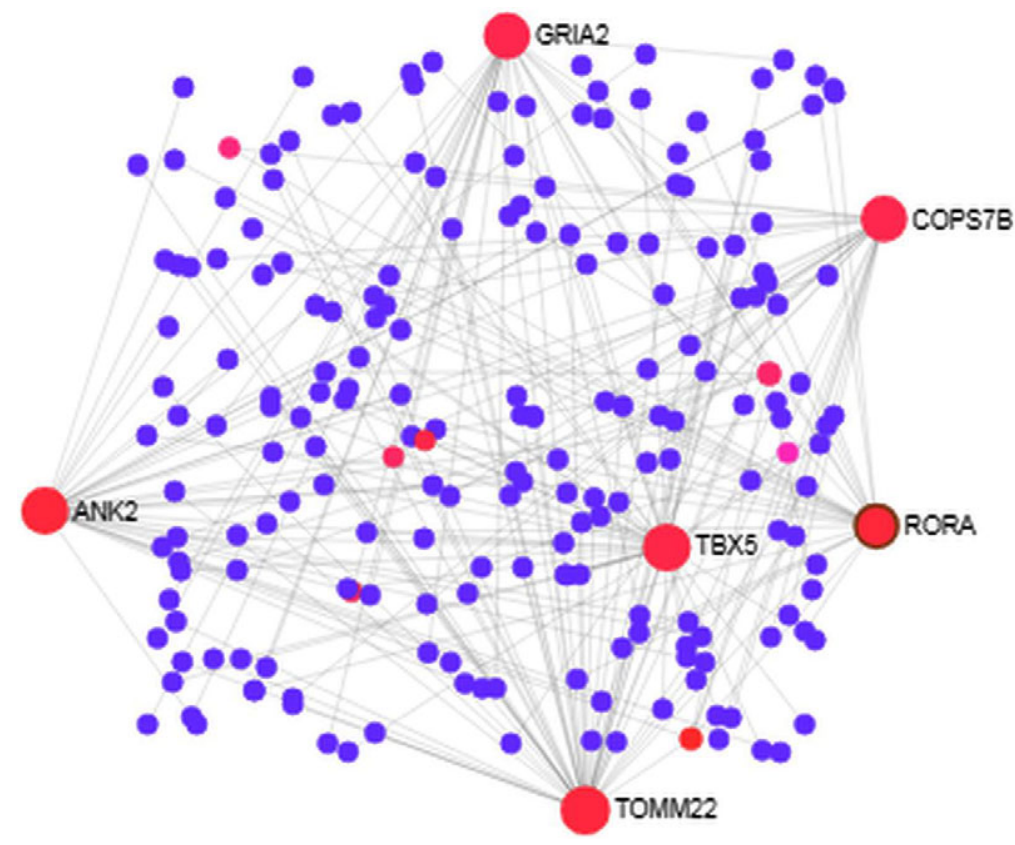

Figure 2. Protein-protein interaction analysis of the Hyper-LGs in Alzheimer's disease. The proteins are represented in nodes (blue and red). Red nodes are marked as hub nodes. The larger node size indicates their degree in topological analysis. Edges in gray show the interactions among interacting proteins in the network.

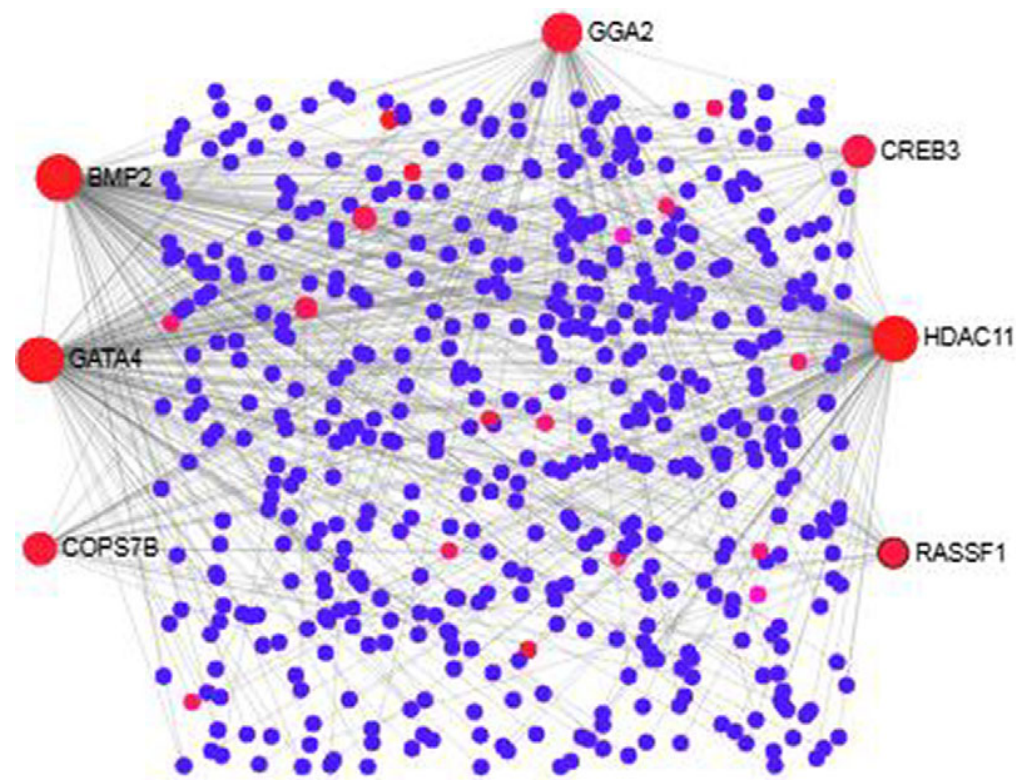

Figure 3. Protein-protein interaction analysis of the Hypo-HGs in Alzheimer's disease. The proteins are represented in nodes (blue and red). Red nodes are marked as hub nodes. The larger node size indicates their degree in topological analysis. Edges in gray show the interactions among interacting proteins in the network.

(Yang et al., 2017) so HDAC11 could be a novel drug target for AD. CREB signaling has been a known link to neurodegenerative disorders and plays many important roles in brain cell functions (Saura \& Valero, 2011). 
Table 2. Transcription factors of methylated-differentially expressed genes related to Alzheimer's disease patient samples.

\begin{tabular}{llll}
\hline Category & Term & Adj. $p$-value & Genes \\
\hline Hyper-LGs & EIF4EBP1 & 0.015 & ANK2;TOMM22 \\
\hline XBP1 & 0.016 & ANK2;R3HDM1;TBX5 \\
\hline NKX2-8 & 0.021 & DGKQ;SLC6A15 \\
\hline MEF2A & 0.030 & GRIA2;CMTM4;SLC6A15;GPR22;ANK2;RORA \\
\hline BCL6 & 0.033 & GRIA2;TMEM67;SLC6A15;GPR22 \\
\hline HNF4A & 0.035 & NTNG1;TMEM67;SLC6A15;ANK2;RORA \\
\hline GATA6 & 0.036 & NTNG1;CA12;ANK2;RORA \\
\hline RELB & 0.038 & KL;DGKQ;SLC6A15;COPS7B \\
\hline THypo-HGS & 0.004 & BMP2;RASSF1;CYHR1;GATA4 \\
\hline HINFP & 0.010 & CREB3;HDAC11;CYHR1;COPS7B;CYBA \\
\hline SP3 & 0.024 & HDAC11;CYHR1;CYBA \\
\hline NR5A1 & 0.027 & CREB3;RASSF1;POLR3E \\
\hline SP1 & 0.028 & HDAC11;CYHR1;CYBA \\
\hline
\end{tabular}

With regard to the Hyper-LGs that we detected, one study detected identified loci in EIF4EBP1 as associated with late onset AD (Nalls et al., 2009). A study has suggested that XBP1 was a risk factor for developing AD (Duran-Aniotz et al., 2017). In addition, XBP1 dysregulation has a profound impact on immune systems, inflammatory response and implicated in complexes diseases including AD (Cisse et al., 2017). A polymorphism in MEF2A could be involved in AD pathogenesis (González et al., 2007). Variation in RELB impacts upon hippocampal function in late onset AD (Xiao et al., 2017). With regard to Hypo-HGs, increased expression of SP3 observed in brains of AD patients (Boutillier et al., 2007). The overexpression of $S P 1$ in AD subjects was reported and suggested as a therapeutic target to help prevent $\mathrm{AD}$ (Citron et al., 2008). However, it should be noted that our study has some limitations in that it does not include data on gene expression or the methylation profiles of the genes in the same brain region. This is due to the lack of any available datasets to investigate these aspects. Thus, although we have uncovered a number of potentially important hub genes and pathways, they require further experimental verifications to establish them as having a definite role $\mathrm{AD}$ pathobiology.

\section{Conclusions}

In the present study, we have analyzed gene expression and DNA methylation profiling in $\mathrm{AD}$. We identified 28 MDEGs and pathway analysis revealed significant enrichment of pathways related to AD pathogenesis. The PPI analysis revealed hub Hyper-LGs of AD included TOMM22, TBX5, ANK2, GRIA2, COPS7B, RORA; such genes for Hypo-HGs included BMP2, GATA4, HDAC11, GGA2, CREB3, RASSF1. Regulatory TFs (EIF4EBP1, XBP1, NKX2-8, MEF2A, BCL6, HNF4A, GATA6, RELB) were identified among the hyper-LGs; similarly we identified TFs (TFAP2C, HINFP, SP1, SP3, NR5A1) influencing Hypo-HGs. Since these are robust candidate genes based on dysregulated methylation, it is possible that these or some significant downstream gene transcription targets of these TFs may be useful for diagnostics (in the case of secreted TFs detectable in the blood) and possibly as treatment targets for $\mathrm{AD}$. The present study improves our understanding of the epigenetic in the pathobiology of AD and identified a number of potential AD biomarkers for further investigation in experimental studies.

Acknowledgements. Authors would like to thank Mr Humayan Kabir Rana for critical reading of the manuscript.

Author contributions. MRR conceived and designed the study; MRR and TI analyzed data; MRR wrote the draft manuscript; EG, JMWQ, and MAM reviewed and edited the manuscript; MAM supervised the project. 
Funding information. This research received no specific grant from any funding agency, commercial or not-for-profit sectors.

Conflict of interest. The authors declare that there is no conflict of interest.

Data availability. Gene expression profiling data with accession GSE4757 and DNA methylation profiling data with accession GSE45775 are publicly available at the Gene Expression Omnibus database (https://www.ncbi.nlm.nih.gov/geo/).

Supplementary Materials. To view supplementary material for this article, please visit http://dx.doi.org/10.1017/exp.2020.65.

\section{References}

Acquaah-Mensah, G. K., Agu, N., Khan, T., \& Gardner, A. (2015). A regulatory role for the insulin- and BDNF-linked RORA in the hippocampus: Implications for Alzheimer's disease. Journal of Alzheimer's. Disease, 44, 827-838. https://doi. org/10.3233/JAD-141731.

Boutillier, S., Lannes, B., Buee, L., Delacourte, A., Rouaux, C., Mohr, M., Bellocq, J.-P., Sellal, F., Larmet, Y., Boutillier, A.L., \& Loeffler, J.-P. (2007). Sp3 and sp4 transcription factor levels are increased in brains of patients with Alzheimer's disease. Neurodegenerative Diseases, 4, 413-423. https://doi.org/10.1159/000107701.

Chouliaras, L., Mastroeni, D., Delvaux, E., Grover, A., Kenis, G., Hof, P. R., Steinbusch, H. W. M., Coleman, P. D., Rutten, B. P. F., \& van den Hove, D. L. A. (2013). Consistent decrease in global DNA methylation and hydroxymethylation in the hippocampus of Alzheimer's disease patients. Neurobiology of Aging, 34, 2091-2099. https://doi.org/10.1016/j.neurobiolaging.2013.02.021.

Cisse, M., Duplan, E., \& Checler, F. (2017). The transcription factor XBP1 in memory and cognition: Implications in Alzheimer's disease. Molecular Medicine, 22, 905-917. https://doi.org/10.2119/molmed.2016.00229.

Citron, B. A., Dennis, J. S., Zeitlin, R. S., \& Echeverria, V. (2008). Transcription factor Sp1 dysregulation in Alzheimer's disease. Journal of Neuroscience Research, 86, 2499-2504. https://doi.org/10.1002/jnr.21695.

Coppieters, N., Dieriks, B. V., Lill, C., Faull, R. L. M., Curtis, M. A., \& Dragunow, M. (2014). Global changes in DNA methylation and hydroxymethylation in Alzheimer's disease human brain. Neurobiology Aging, 35, 1334-1344. https://doi. org/10.1016/j.neurobiolaging.2013.11.031.

Cordner, R. D., Friend, L. N., Mayo, J. L., Badgley, C., Wallmann, A., Stallings, C. N., Young, P. L., Miles, D. R., Edwards, J. G., \& Bridgewater, L. C. (2017). The BMP2 nuclear variant, nBMP2, is expressed in mouse hippocampus and impacts memory. Scientific Reports, 7, 46464. https://doi.org/10.1038/srep46464.

Dunckley, T., Beach, T. G., Ramsey, K. E., Grover, A., Mastroeni, D., Walker, D. G., LaFleur, B. J., Coon, K. D., Brown, K. M., Caselli, R., Kukull, W., Higdon, R., McKeel, D., Morris, J. C., Hulette, C., Schmechel, D., Reiman, E. M., Rogers, J., \& Stephan, D. A. (2006). Gene expression correlates of neurofibrillary tangles in Alzheimer's disease. Neurobiology Aging, 27, 1359-1371. https://doi.org/10.1016/j.neurobiolaging.2005.08.013.

Duran-Aniotz, C., Cornejo, V. H., Espinoza, S., Ardiles, A. O., Medinas, D. B., Salazar, C., Foley, A., Gajardo, I., Thielen, P., Iwawaki, T., Scheper, W., Soto, C., Palacios, A. G., Hoozemans, J. J. M., \& Hetz, C. (2017). IRE1 signaling exacerbates Alzheimer's disease pathogenesis. Acta Neuropathologica, 134, 489-506. https://doi.org/10.1007/s00401-017-1694-x.

Garranzo-Asensio, M., San Segundo-Acosta, P., Martinez-Useros, J., Montero-Calle, A., Fernandez-Acenero, M. J., Haggmark-Manberg, A., Pelaez-Garcia, A., Villalba, M., Rabano, A., Nilsson, P., \& Barderas, R. (2018). Identification of prefrontal cortex protein alterations in Alzheimer's disease. Oncotarget, 9, 10847-10867. https://doi.org/10.18632/ oncotarget.24303.

Gomez Ravetti, M., Rosso, O. A., Berretta, R., \& Moscato, P. (2010). Uncovering molecular biomarkers that correlate cognitive decline with the changes of hippocampus' gene expression profiles in Alzheimer's disease. PLoS One, 5, e10153. https://doi.org/10.1371/journal.pone.0010153.

González, P., Alvarez, V., Menéndez, M., Lahoz, C. H., Martínez, C., Corao, A. I., Calatayud, M. T., Peña, J., García-Castro, M., \& Coto, E. (2007). Myocyte enhancing factor-2A in Alzheimer's disease: Genetic analysis and association with MEF2Apolymorphisms. Neuroscience Letters, 411, 47-51. https://doi.org/10.1016/j.neulet.2006.09.055.

Higham, J. P., Malik, B. R., Buhl, E., Dawson, J., Ogier, A. S., Lunnon, K., \& Hodge, J. J. L. (2018). Mis-expression of the Alzheimer's disease associated gene Ankyrin causes memory loss and shortened lifespan in Drosophila. bioRxiv 423129. https://doi.org/10.1101/423129

Hu, W., Wang, Z., \& Zheng, H. (2018). Mitochondrial accumulation of amyloid beta (Abeta) peptides requires TOMM22 as a main Abeta receptor in yeast. Journal of Biological Chemistry, 293, 12681-12689. https://doi.org/10.1074/jbc.RA118.002713.

Kawalia, S. B., Raschka, T., Naz, M., de Matos Simoes, R., Senger, P., \& Hofmann-Apitius, M. (2017). Analytical strategy to prioritize Alzheimer's disease candidate genes in gene regulatory networks using public expression data. Journal of Alzheimer's Diseases, 59, 1237-1254. https://doi.org/10.3233/JAD-170011.

Kuleshov, M. V., Jones, M. R., Rouillard, A. D., Fernandez, N. F., Duan, Q., Wang, Z., Koplev, S., Jenkins, S. L., Jagodnik, K. M., \& Lachmann, A. (2016). Enrichr: A comprehensive gene set enrichment analysis web server 2016 update. Nucleic Acids Research, 44, W90-W97. 
Mueller, K. A., Glajch, K. E., Huizenga, M. N., Wilson, R. A., Granucci, E. J., Dios, A. M., Tousley, A. R., Iuliano, M., Weisman, E., LaQuaglia, M. J., DiFiglia, M., Kegel-Gleason, K., Vakili, K., \& Sadri-Vakili, G. (2018). Hippo signaling pathway dysregulation in human Huntington's disease brain and neuronal stem cells. Scientific Reports, 8, 11355. https://doi. org/10.1038/s41598-018-29319-4.

Nalls, M. A., Guerreiro, R. J., Simon-Sanchez, J., Bras, J. T., Traynor, B. J., Gibbs, J. R., Launer, L., Hardy, J., \& Singleton, A. B. (2009). Extended tracts of homozygosity identify novel candidate genes associated with late-onset Alzheimer's disease. Neurogenetics, 10, 183-190. https://doi.org/10.1007/s10048-009-0182-4.

O’Neill, J., Boccara, C. N., Stella, F., Schönenberger, P., \& Csicsvari, J. (2017). Superficial layers of the medial entorhinal cortex replay independently of the hippocampus. Science, 355, 184-188. https://doi.org/10.1126/science.aag2787.

Rahman, M. R., Islam, T., Zaman, T., Shahjaman, M., Karim, M. R., Huq, F., Quinn, J. M. W., Holsinger, R. M. D., \& Moni, M. A. (2020). Identification of biomarkers and pathways to identify novel therapeutic targets in Alzheimer's disease: Insights from a systems biomedicine perspective. Genomics, 112, 1290-1299. https://doi.org/10.1016/j.ygeno.2019.07.018.

Rusina, R., Sheardova, K., Rektorova, I., Ridzon, P., Kulist'ak, P., \& Matej, R. (2007). Amyotrophic lateral sclerosis and Alzheimer's disease--clinical and neuropathological considerations in two cases. European Journal of Neurology, 14, 815-818. https://doi.org/10.1111/j.1468-1331.2007.01759.x.

Sanchez-Mut, J. V., \& Graff, J. (2015). Epigenetic alterations in Alzheimer's disease. Frontiers in Behavioral Neuroscience, 9 , 347. https://doi.org/10.3389/fnbeh.2015.00347.

Saura, C. A., \& Valero, J. (2011). The role of CREB signaling in Alzheimer's disease and other cognitive disorders. Reviews in the Neurosciences, 22, 153-169. https://doi.org/10.1515/RNS.2011.018.

Semick, S. A., Bharadwaj, R. A., Collado-Torres, L., Tao, R., Shin, J. H., Deep-Soboslay, A., Weiss, J. R., Weinberger, D. R., Hyde, T. M., Kleinman, J. E., Jaffe, A. E., \& Mattay, V.S. (2019). Integrated DNA methylation and gene expression profiling across multiple brain regions implicate novel genes in Alzheimer's disease. Acta Neuropathologica, 137, 557-569. https://doi. org/10.1007/s00401-019-01966-5.

Stoccoro, A., \& Coppede, F. (2018). Role of epigenetics in Alzheimer's disease pathogenesis. Neurodegenerative Disease Management, 8, 181-193. https://doi.org/10.2217/nmt-2018-0004.

Szklarczyk, D., Morris, J. H., Cook, H., Kuhn, M., Wyder, S., Simonovic, M., Santos, A., Doncheva, N. T., Roth, A., Bork, P., Jensen, L. J., \& Von Mering, C. (2017). The STRING database in 2017: Quality-controlled protein-protein association networks, made broadly accessible. Nucleic Acids Research, 45, D362-D368. https://doi.org/10.1093/nar/gkw937.

von Bernhardi, R., Cornejo, F., Parada, G. E., \& Eugenin, J. (2015). Role of TGFbeta signaling in the pathogenesis of Alzheimer's disease. Frontiers in Cellular Neuroscience, 9, 426. https://doi.org/10.3389/fncel.2015.00426.

Xia, J., Gill, E. E., \& Hancock, R. E. W. (2015). NetworkAnalyst for statistical, visual and network-based meta-analysis of gene expression data. Nature Protocols, 10, 823.

Xiao, E., Chen, Q., Goldman, A. L., Tan, H. Y., Healy, K., Zoltick, B., Das, S., Kolachana, B., Callicott, J. H., Dickinson, D., Berman, K. F., Weinberger, D. R., \& Mattay, V. S. (2017). Late-onset Alzheimer's disease polygenic risk profile score predicts hippocampal function. Biological Psychiatry: Cognitive Neuroscience and Neuroimaging, 2, 673-679. https://doi. org/10.1016/j.bpsc.2017.08.004.

Yang, S., Zhang, R., Wang, G., \& Zhang, Y. (2017). The development prospection of HDAC inhibitors as a potential therapeutic direction in Alzheimer's disease. Translational Neurodegeneration, 6, 19. https://doi.org/10.1186/s40035-0170089-1.

Cite this article: Rahman MR, Islam T, Gov E, Quinn JMW, Moni MA (2021). Identifying the function of methylated genes in Alzheimer's disease to determine epigenetic signatures: a comprehensive bioinformatics analysis Experimental Results, 2 , e9, 1-13. https://doi.org/10.1017/exp.2020.65 


\title{
Peer Reviews
}

\author{
Reviewing editor: Dr. Sourav Kolay \\ UT Southwestern, 5323 Harry Hines Blvd, Dallas, Texas, United States, 75390-9096
}

This article has been accepted because it is deemed to be scientifically sound, has the correct controls, has appropriate methodology and is statistically valid, and has been sent for additional statistical evaluation and met required revisions.

doi:10.1017/exp.2020.65.pr1

Review 1: Identifying the Function of Methylated Genes in Alzheimer's Disease to Determine Epigenetic Signatures: A Comprehensive Bioinformatics Analysis

Reviewer: Dr. Md. Ataur Rahman

Korea Institute of Science and Technology, Neuroscience Centre

Date of review: 08 November 2020

(C) The Author(s) 2021. Published by Cambridge University Press This is an Open Access article, distributed under the terms of the Creative Commons Attribution licence (http://creativecommons.org/licenses/by/4.0/), which permits unrestricted re-use, distribution, and reproduction in any medium, provided the original work is properly cited.

Conflict of interest statement. Reviewer declares none

Comments to the Author: Title: Identifying the Function of Methylated Genes in Alzheimer's Disease to Determine Epigenetic Signatures: A Comprehensive Bioinformatics Analysis

In the present manuscript the authors apply a variety of bioinformatics approaches to identify differentially methylated genes and differentially expressed genes from available datasets on Alzheimer's disease to understand their biological pathways and interconnections. This manuscript is very interesting and advance epigenetics filed of Alzheimer's disease. The manuscript is clearly written and the results are well presented, but I suggest some minor revisions:

1) The manuscript should be improved in the level of detail and description of both materials and method and results, including figure captions.

2) The significance of the hub genes should be stressed in the discussion section.

3) The discussion section should be concise

4) Please check for grammar and typos. (for example, interaction is misspelt in discussion).

\section{Score Card}

Presentation

Is the article written in clear and proper English? (30\%)

Is the data presented in the most useful manner? (40\%)

Does the paper cite relevant and related articles appropriately? (30\%) 
Does the abstract correctly embody the content of the article? (25\%)

Does the introduction give appropriate context? (25\%)

Is the objective of the experiment clearly defined? (25\%)

Analysis

Does the discussion adequately interpret the results presented? (40\%)

Is the conclusion consistent with the results and discussion? (40\%)

Are the limitations of the experiment as well as the contributions of the experiment clearly outlined? $(20 \%)$ 


\section{Review 2: Identifying the Function of Methylated Genes in Alzheimer's Disease to Determine Epigenetic Signatures: A Comprehensive Bioinformatics Analysis}

Reviewer: Dr. Kichang Kwak

Date of review: 14 November 2020

(C) The Author(s) 2021. Published by Cambridge University Press This is an Open Access article, distributed under the terms of the Creative Commons Attribution licence (http://creativecommons.org/licenses/by/4.0/), which permits unrestricted re-use, distribution, and reproduction in any medium, provided the original work is properly cited.

Conflict of interest statement. Reviewer declares none

Comments to the Author: The paper describes the application of gene expression data to identify differentially expressed genes and DNA methylation data to identify differentially methylated genes to identify overlapping methylated differentially expressed genes to provide novel insights in AD pathology. Overall, the paper is fairly organized. But before publication, the authors should address the following points:

1. Add the reference for first sentence "Alzheimer's disease (AD) is a neurodegenerative " in introduction (page 3).

2. Rephrase the sentence "In this study, we analyzed gene expression " in objective (page 4).

3. Why did you use a threshold value for degree as 20 ? Clarify a threshold value for degree (page 6).

4. What is the regulatory TFs? Define "regulatory TFs" (page 6).

5. How did you get the adjusted p value? Clarify the statistical methods, for example multiple comparison correction or covariates.

6. Make sure to discuss the limitations of this study.

7. Proof-read the entire text for minor grammatical errors, especially abbreviations.

\section{Score Card}

Presentation

Is the article written in clear and proper English? (30\%)

Is the data presented in the most useful manner? (40\%)

Does the paper cite relevant and related articles appropriately? (30\%)

Context

Does the title suitably represent the article? (25\%)

Does the abstract correctly embody the content of the article? (25\%)

Does the introduction give appropriate context? (25\%)

Is the objective of the experiment clearly defined? (25\%) 
Analysis

Is the conclusion consistent with the results and discussion? (40\%)

Are the limitations of the experiment as well as the contributions of the experiment clearly outlined? (20\%) 